\title{
Infections of Soil-Transmitted Helminth in Refugees from North Korea
}

\author{
Young-II Lee', Min Seo², Suk-Bae Kim,* \\ 'Department of Anatomy, College of Medicine, Dankook University, Cheonan 31116, Korea; ${ }^{2}$ Department of Parasitology, College of Medicine, \\ Dankook Unverisity, Cheonan 31116, Korea; ${ }^{3}$ Division of Gastroenterology, Department of Internal Medicine, College of Medicine, Dankook \\ University, Cheonan 31116, Korea
}

\begin{abstract}
Soil-transmitted helminthiases (STH) are now no longer public health problems in the Republic of Korea (South Korea), but their status are unavailable in the residents of North Korea (NK) despite the expectation of large scale traffic and future reunification of the Korean Peninsula. A total of 20 female refugees from NK who had been admitted to the Division of Gastroenterology, Dankook University Hospital, were subjected in this study. Among them, 15 refugees were examined by the colonoscopy and 10 ones were examined with the stool examination (formalin-ether sedimentation). Both diagnostic methods were commonly adopted in 5 patients. Eggs of Trichuris trichiura were detected in 7 out of 10 refugees in the stool examination. In the colonoscopy, T. trichiura worms were found in $6(40.0 \%)$ out of 15 refugees. Total 9 (45.0\%) peoples were confirmed to be infected with human whipworms. Additionally, 1 case of clonorchiasis was diagnosed in the stool examination and a worm of Ascaris lumbricoides was discovered from a trichuriasis case. These findings suggested that STH is highly prevalent in NO, in which living conditions are not so good in the aspect of general hygiene and medical care.
\end{abstract}

Key words: Trichuris trichiura, Clonorchis sinensis, Ascaris lumbricoides, helminth, refugees, colonoscopy, stool examination, North Korea

Parasite-infection rates have been dramatically reduced around the world by the introduction of chemical fertilizers and the continuous development of infectious-disease management systems [1]. Whereas there were no differences in the parasite-infection statuses of North and South Korea in the 1950s, the situation has been altered dramatically over the intervening decades with the successful eradication in South Korea of intestinal helminthiases [2]. Although some food-borne and zoonotic parasite infections along with malaria are still reported frequently, soil-transmitted parasites have been well controlled in South Korea [3]. Soil-transmitted helminthiases (STH), however, remain prevalent in some areas of North Korea (NK) and among North Korean refugees who manage to escape to the south $[4,5]$. Whereas a clear understanding of the health status of NK is mandatory for STH prevention and control measures, this objective is hampered by the lack of reliable data [6]. Even in South Korea these days, the parasite-infection

\footnotetext{
- Received 31 October 2017, revised 15 May 2018, accepted 2 June 2018.

*Corresponding author (dryakson@hanmail.net)

(C) 2018, Korean Society for Parasitology and Tropical Medicine

This is an Open Access article distributed under the terms of the Creative Commons

Attribution Non-Commercial License (http://creativecommons.org/licenses/by-nc/4.0)

which permits unrestricted non-commercial use, distribution, and reproduction in any

medium, provided the original work is properly cited.
}

prevalence is increasing, due to international marriage, immigration, overseas traveling, and increased importation of foodstuffs $[7,8]$.

All of these issues notwithstanding, exact and detailed monitoring of infectious disease status in NK is needed now more than ever before; the number of North Korean refugees escaping to South Korea is at a constant rate, and a large-scale traffic between North and South Korea is also expected at any future. However, surveillance of parasite-infection patterns and status in NK currently are restricted to some limited-scale borderline areas. Therefore as a part of continuous data collection on STH in NK, the purpose of this study is to evaluate status of intestinal helminth infections among the refugees from NK which is practically achievable surveillance at present.

In the present study, a total of 20 subjects were admitted to the Division of Gastroenterology, Department of Internal Medicine, Dankook University Hospital, due to various symptoms occurring between October 2009 and February 2013. All of them were female refugees from $\mathrm{NK}$, aged between 17 and 56. Some of them were being managed or evaluated for certain clinical conditions such as liver mass, chronic hepatitis $\mathrm{C}$, or pulmonary tuberculosis. Along with history taking and routine diagnostic workups, all of the subjects were examined by 



Fig. 1. Recovered worms from the North Korean refugees. (A-C) The adult worms of Trichuris trichiura, removed by colonoscopy. (D) An adult worm of Ascaris lumbricoides, removed by gastroscopy.

colonoscopy or stool examination to confirm their parasite-infection status. Among the 20 patients, 15 were examined by colonoscopy and the stools of 10 were examined by microscopy following formalin-ether sedimentation technique. Therefore, 5 patients were examined by both colonoscopy and stool. Additionally, gastroscopic examinations were performed on patients with epigastric or abdominal pain.

A total 10 out of 20 subjects (50.0\%) were found to be in- fected by intestinal helminthes: 9 cases (45.0\%) were diagnosed as Trichuris trichiura infection and 1 case (5.0\%) as Clonorchis sinensis. Among the 15 patients examined by colonoscopy, adult worms of T. trichiura were identified in 6 cases (40\%). In the stool examinations performed for 10 patients, there were 7 (70.0\%) positive results: 6 of them were T. trichiura cases and only 1 was Clonorchis sinensis case. Among the 5 patients of trichuriasis who were examined by both colonos- 
Table 1. Individual data on the patients with trichuriasis ${ }^{a}$

\begin{tabular}{|c|c|c|c|c|c|c|}
\hline No. & Age & Complaints & Diagnosis & Stool & $\begin{array}{l}\text { Colonoscopy } \\
\text { (No. of worms) }\end{array}$ & Note \\
\hline 1 & 42 & liver mass & liver hemangioma & + & 1 & \\
\hline 2 & 34 & abdominal pain & liver cirrhosis & + & 1 & A. lumbricoides \\
\hline 3 & 47 & epigastric pain & acute gastroenteritis & + & 6 & by gastroscopy \\
\hline 4 & 42 & itching & liver cirrhosis & + & 5 & \\
\hline 5 & 24 & pulmonary TB & pulmonary TB & + & $N D^{b}$ & \\
\hline 6 & 36 & known chronic hepatitisC & chronic hepatitis C & - & 1 & \\
\hline 7 & 17 & $R \cup Q$ pain & liver abscess & + & - & \\
\hline 8 & 45 & $R \cup Q$ pain & chronic hepatitis C & ND & 32 & \\
\hline 9 & 56 & cough, sputum & pulmonary TB & + & - & \\
\hline
\end{tabular}

${ }^{a}$ All the patients were female.

${ }^{\text {bNot done. }}$

copy and stool examination, 3 patients were identified by both methods (Fig. 1) while 2 other patients were identified by either colonoscopy or stool examination. Among the cases of trichuriasis in this study, 1 case was confirmed while evaluating a liver mass and 2 other cases were confirmed while managing chronic hepatitis $\mathrm{C}$ and pulmonary tuberculosis. Interestingly in a patient with trichuriasis, adult worm of Ascaris lumbricoides was also detected in the duodenum by gastroscopy. The information on the trichuriasis patients was summarized in Table 1. All of the patients were treated with albendazole or praziquantel according to the specific parasite by which they were infected.

These results indicate that the parasite-infection rate of refugees from NK is still considerably high and that most cases are typical soil-transmitted helminth infection reflective of poor general hygiene and inadequate medical care. As shown in the results of this study, the majority of parasite positive cases were confirmed as T. trichiura infection. The high prevalence of trichuriasis in this study is in concordance with previous studies $[4,5]$. According to Lee et al. [9], a refugee from NK diagnosed by colonoscopy was determined to have most probably been infected before leaving NK, as had been suffering the typical symptoms of intestinal helminthiasis while still living there.

Since soil-transmitted helminth infection had already eradicated in South Korea [2], probably all of the patients confirmed as suffering trichuriasis in this study had been infected while living in NK. Trichuriasis is often accompanied by hookworm infection, which accelerates the onset of iron-deficiency anemia. Therefore, the possibility of our subjects harboring hookworm infection along with trichuriasis still remains. Indeed, among the trichuriasis-confirmed cases in this study, 1 individual's stool analysis did not reveal any eggs. Application of more sensitive and reliable diagnostic tools such as ELISA (enzyme-linked immunosorbent assay) [10-12] are needed for the accumulation of more accurate data. An additional limitation of this study is the fact that the number of subjects was relatively small and limited to female. Along with the majority trichuriasis cases confirmed in the present study, 1 case of $C$. sinensis infection was detected by stool examination. This patient possibly had been infected before leaving NK, which is located between 2 known endemic zones; South Korea and Northeast China [13]. Also, most refugees reside in China, Mongolia, and Southeast Asian countries before proceeding to South Korea, which migration routes expose them to risks of food-borne helminthiases including $C$. sinensis. Interestingly in the case of cysticercosis, the positive ratio of this metacestode among refugees is higher than that among North Korean residents, whose migration route, tellingly includes countries that are endemic for cysticercosis [14,15].

In summary, as the present study was not based on local surveillance, they might not reflect the real situations in NK. However, continued collection of data on STH among refugees themselves, seems the most practical and prudent course of action at present. The number of refugees concurrently is increasing, and a large scale influx of population is expected both during and for some time after any future process of reunification process. This is why the collection of data on the parasiteinfection statuses of refugees is so important.

\section{CONFLICT OF INTEREST}

All authors declare that they have no conflict of interest to this study. 


\section{REFERENCES}

1. Hong ST. Changes of anti-Clonorchis sinensis IgG antibody in serum after praziquantel treatment in human clonorchiasis. Korean J Parasitol 1988; 26: 1-8.

2. Lee SH. Forty years history of the Korea Association of Health Promotion - Control of parasitic diseases and the Korea Association of Health Promotion - Health News 2005; 6-11.

3. Hong ST, Chai JY, Choi MH, Huh S, Rim HJ, Lee SH. A successful experience of soil-transmitted helminth control in the Republic of Korea. Korean J Parasitol 2006; 44: 177-185.

4. Li S, Shen C, Choi MH, Bae YM, Yoon H, Hong ST. Status of intestinal helminthic infections of borderline residents in North Korea. Korean J Parasitol 2006; 44: 265-268.

5. Shen C, Li S, Zheng S, Choi MH, Bae YM, Hong ST. Tissue parasitic helminthiases are prevalent at Cheongin, North Korea. Korean J Parasitol 2007; 45: 139-144.

6. McCurry J. North Korea's health system in disarray. Lancet 2010; 376: 318.

7. Lee SH. Transition of parasitic diseases in Korea. J Korean Med Assoc 2007; 50: 937-945.

8. Kim HS, Lee JH, Choi YH, Kim JH, Son HJ, Rhee PL, Kim JJ, Lee MK, Rhee JC. Examination for helminth eggs and protozoan cysts in fecal samples from healthy Korean adults, 2000-2006. Korean J Med 2009; 77: 741-749.
9. Lee SH, Kwon JE, Cheong YS. Two cases of Trichuris trichiura infection diagnosed by colonoscopy. Korean J Fam Med 2010; 31: 622-629.

10. Kim H, Kim SI, Cho SY. Serological diagnosis of human sparganosis by means of micro-ELISA. Korean J Parasitol 1984; 22: 222-228.

11. Cho SY, Kim SI, Kang SY, Choi DY, Suk JS, Choi KS, Ha YS, Chung CS, Myung HJ. Evaluation of enzyme-linked immunosorbent assay in serological diagnosis of human neurocysticercosis using paired samples of serum and cerebrospinal fluid. Korean J Parasitol 1986; 24: 25-41.

12. Hong ST. Current status of parasitic infection in Korea. J Korean Med Assoc 1998; 41: 737-745.

13. Coordinating Office of the National Survey on the Important Human Parasitic Diseases. A national survey on current status of the important parasitic diseases in human population. Chinese J Parasitol Parasitic Dis 2005; 23: 332-340 (in Chinese).

14. Chung JY, Eom KS, Yang Y, Li X, Feng Z, Rim HJ, Cho SY, Kong Y. A seroepidemiological survey of Taenia solium cysticercosis in Nabo, Guangxi Zhuang Autonomous Region, China. Korean J Parasitol 2005; 43: 135-139.

15. Somers R, Dorny P, Nguyen VK, Dang TC, Goddeeris B, Craig PS, Vercruysse J. Taenia solium taeniasis and cysticercosis in three communities in north Vietnam. Trop Med Int Health 2006; 11: 65-72. 